\title{
A Case of Non-Resolving MEK Inhibitor-Associated Retinopathy
}

\author{
John R. Chancellor ${ }^{a}$ David A. Kilgore ${ }^{b} \quad$ Ahmed B. Sallam ${ }^{a}$ \\ Richard C. Allen ${ }^{c}$ Sami H. Uwaydat ${ }^{a}$ \\ Jones Eye Institute, Department of Ophthalmology, University of Arkansas for Medical \\ Sciences, Little Rock, AR, USA; bUniversity of Arkansas for Medical Sciences, College of \\ Medicine, Little Rock, AR, USA; 'Section of Ophthalmology, Department of Head and Neck \\ Surgery, University of Texas MD Anderson Cancer Center, Houston, TX, USA
}

\section{Keywords}

MEK inhibitor · Paraneoplastic vitelliform retinopathy · Melanoma

\begin{abstract}
The mitogen-activated kinase pathway plays an important role in cell survival, and its dysregulation is associated with cancers such as melanoma. Drugs designed to target this pathway have been associated with serous retinal detachments in a new entity termed MEK inhibitorassociated retinopathy (MEKAR). MEKAR has classically been described as self-limiting, with serous fluid often resolving without discontinuation of the drug. We present a case in which a patient undergoing treatment for metastatic melanoma with lacnotuzumab, a macrophage colony-stimulating factor inhibitor that blocks an upstream component of the mitogen-activated protein kinase pathway, developed serous retinopathy that did not resolve despite drug discontinuation.

\section{Introduction}

MEK inhibitors such as binimetinib, trametinib, and dabrafenib have exhibited effectiveness in the treatment of melanoma, but their use is associated with serous detachments of the neurosensory retina, termed MEK inhibitor-associated retinopathy (MEKAR) [1, 2]. MEKAR is 
classically self-limiting, with serous fluid resolving within 1-2 months even without drug discontinuation [2, 3]. In addition, metastatic melanoma itself can result in serous retinopathy with detachments of the retinal pigment epithelium (RPE) and neurosensory retina due to a cross-reaction by circulating autoantibodies [4]. This type of retinopathy is termed paraneoplastic vitelliform retinopathy [4]. We present a case of a patient with metastatic melanoma treated with lacnotuzumab, a mitogen-activated protein kinase (MAPK) pathway inhibitor, who developed non-resolving serous detachments (Fig. 1a).

\section{Case Report}

A 54-year-old male presented to ophthalmology 18 months after receiving the diagnosis of colorectal melanoma. He had been treated with multiple anti-cancer medications prior to beginning a new study protocol with lacnotuzumab and spartalizumab.

Four days following initiation of lacnotuzumab and spartalizumab, the patient reported bilateral blurred vision. Examination showed corrected visual acuity of 20/70 OD and 20/20 OS. Pupils, intraocular pressure, and anterior segment examinations were normal. Fundus examination demonstrated bilateral serous macular edema, normal optic nerves, vessels, and periphery (Fig 1b). Optical coherence tomography (OCT) showed significant subretinal and intraretinal fluid (Fig. 2d). He was diagnosed with MEKAR, the drugs were discontinued, and he was removed from the study protocol.

Two months later, he reported worsening of vision bilaterally. Corrected visual acuity was $20 / 60$ OD and 20/30 + 1 OS. OCT showed resolution of intraretinal fluid but persistence of subretinal fluid (Fig. 2e). Fluorescein angiography demonstrated early and late hypo-fluorescence in the areas of serous fluid accumulation (Fig. 1d, e). Indocyanine green angiography demonstrated normal choroidal filling with hypocyanescence centrally (Fig. 1c). The following month, his vision continued to worsen to 20/100 OD and 20/40-1 OS. OCT demonstrated persistent serous macular detachment and subretinal fluid (Fig. 2f).

Seven months after the patient's treatment with lacnotuzumab and spartalizumab and onset of symptoms, his corrected visual acuity was 20/200 OD and 20/70 + 1 OS. Examination and autofluorescence showed persistent serous macular detachment with subretinal accumulation of exudates and vitelliform material in a gravity-dependent manner (Fig. 2a, b, c); OCT continued to demonstrate subretinal fluid (Fig. 2g). The patient was scheduled for follow-up in 2 months but unfortunately his disease progressed, and he passed away 7 weeks after this ophthalmology visit.

\section{Discussion/Conclusion}

Lacnotuzumab inhibits macrophage colony-stimulating factor (M-CSF), which is an upstream component of the MAPK pathway (Fig. 1a). Spartalizumab is directed against the negative immunoregulatory human cell surface receptor programmed death-1 and has not been associated with serous retinopathy [5]. Our patient developed visual symptoms and bilateral serous detachments 4 days after starting lacnotuzumab, consistent with a diagnosis of MEKAR $[2,3]$. Typically, fluid accumulation is self-limited, regardless of discontinuation of the drug, with a mean time to resolution of 47.4 days [2]. However, our patient continued to display serous detachments for 7 months (216 days) and remained symptomatic until he passed away (266 days). 


\section{Case Reports in Ophthalmology}

This case is of particular interest due to its unusually long duration and lack of improvement following discontinuation of lacnotuzumab, which suggests one of several possible conclusions. First, lacnotuzumab works upstream of the MEK inhibitors binimetinib, trametinib, and dabrafenib that have been implicated in previous MEKAR cases. Targeting of the upstream M-CSF molecule instead of the MEK protein in the MAPK pathway may lead to a prolonged and non-resolving serous MEKAR with eventual accumulation of vitelliform material. This could account for the large unifocal area of subretinal fluid rather than the classic dome, caterpillar, wavy, or splitting patterns noted previously [2]. Second, the patient may have developed a concomitant paraneoplastic vitelliform retinopathy at some time after the development of MEKAR and prior to its resolution [4].

While it is possible the patient developed a concomitant paraneoplastic vitelliform retinopathy, we believe this to be very unlikely [4]. The patient had a diagnosis of colorectal melanoma for 18 months without any symptomatic vision changes. Then, only 4 days after starting lacnotuzumab, the patient had a decrease in vision, and a serous retinopathy was noted on OCT.

Attachment of the neurosensory retina to the pigment epithelium and choriocapillaris requires a balance of fluid flow between the vitreous and underlying choroid [6]. Serous retinal detachment typically occurs via one of two mechanisms: increased permeability of choroidal blood vessels or decreased ability of the RPE to maintain the fluid gradient [6]. Again, given the timing from initiation of lacnotuzumab to the development of serous fluid, we believe that the retinal detachment was likely a result of toxicity to the RPE and decreased pump function. Given the patient's state of poor health with metastatic cancer, it is possible his pigment epithelium was unable to recover from the initial insult as has been seen with other cases of MEKAR [2, 3]. It may also be possible that lacnotuzumab exhibits more severe toxicity to the RPE than the MEK inhibitors previously described.

To our knowledge, this is the first reported case of M-CSF-induced serous retinopathy that persisted despite discontinuation of treatment. When treating patients with a MEK inhibitor, physicians should be aware of the possible association of lacnotuzumab with chronic serous macular detachment.

\section{Statement of Ethics}

The subject has given his informed consent and the study protocol conforms to the institute's IRB requirements.

\section{Disclosure Statement}

The authors have no conflicts of interest to declare.

\section{Funding Sources}

This work was sponsored in part by an endowment from the Martha Wood Bentley Chair in Ophthalmology. 


\section{Author Contributions}

J.R.C., D.A.K., A.B.S., R.C.A., and S.H.U. contributed to the design, acquisition of data, research, drafting, and revision of the manuscript. All authors reviewed and approved the final manuscript for publication.

\section{References}

1 Akinleye A, Furqan M, Mukhi N, Ravella P, Liu D. MEK and the inhibitors: from bench to bedside. J Hematol Oncol. 2013 Apr;6(1):27.

2 Francis JH, Habib LA, Abramson DH, Yannuzzi LA, Heinemann M, Gounder MM, et al. Clinical and morphologic characteristics of MEK inhibitor-associated retinopathy: differences from central serous chorioretinopathy. Ophthalmology. 2017 Dec;124(12):1788-98.

3 Weber ML, Liang MC, Flaherty KT, Heier JS. Subretinal Fluid Associated With MEK Inhibitor Use in the Treatment of Systemic Cancer. JAMA Ophthalmol. 2016 Aug;134(8):855-62.

4 Aronow ME, Adamus G, Abu-Asab M, Wang Y, Chan CC, Zakov ZN, et al. Paraneoplastic vitelliform retinopathy: clinicopathologic correlation and review of the literature. Surv Ophthalmol. 2012 Nov;57(6):558-64.

5 Bitton K, Michot JM, Barreau E, Lambotte O, Haigh O, Marabelle A, et al. Prevalence and clinical patterns of ocular complications associated with anti-PD-1/PD-L1 anticancer immunotherapy. Am J Ophthalmol. 2019 Jun;202:109-117.

6 Jampol LM, Weinreb R, Yannuzzi L. Involvement of corticosteroids and catecholamines in the pathogenesis of central serous chorioretinopathy: a rationale for new treatment strategies. Ophthalmology. 2002 Oct;109(10):1765-6. 


\section{Case Reports in Ophthalmology}
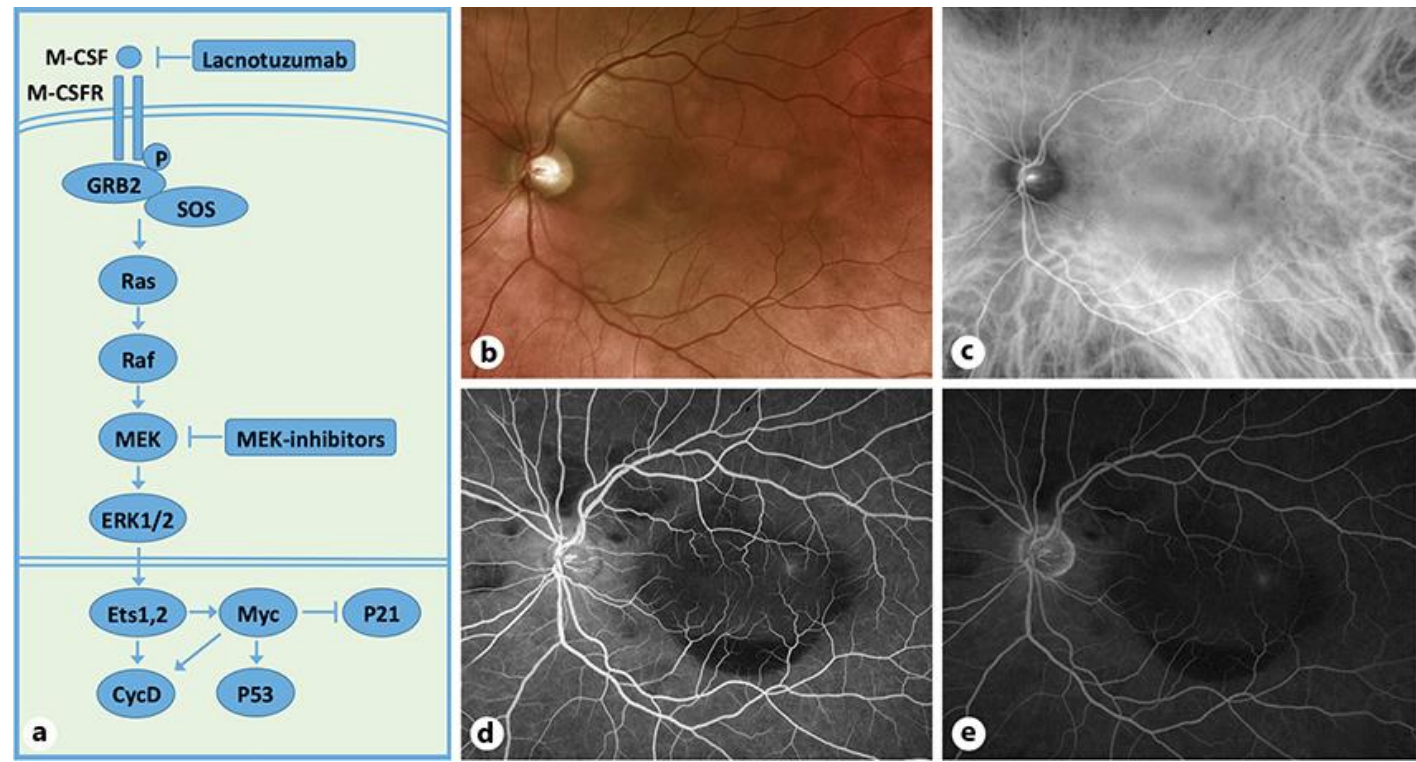

Fig. 1. a Schematic of the mitogen-activated kinase pathway including M-CSF, Ras, MEK, and ERK signaling molecules. Lacnotuzumab is an inhibitor of M-CSF, which is upstream up the MEK inhibitors. b Optos (Optos Inc., USA) fundus photo of the left eye demonstrating subretinal fluid blunting the foveal reflex. c Indocyanine green angiography demonstrated normal levels of fluorescence with blunting of the signal centrally under the site of serous fluid accumulation. Early (0:48) (d) and late (e) (10:50) fluorescein angiography demonstrated early and late hypofluorescence in the areas of serous fluid accumulation without any associated retinal hyperfluorescence.
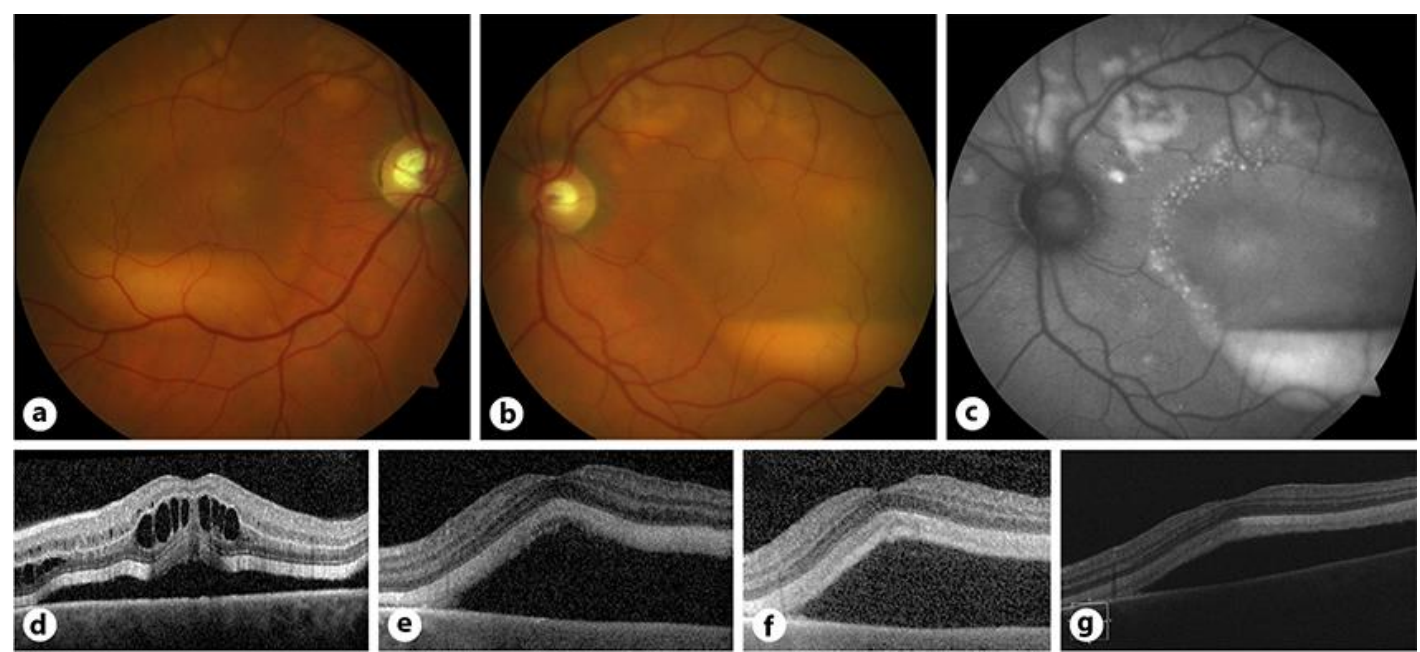

Fig. 2. a, b Color fundus photographs of the right and left eyes demonstrating persistence of subretinal fluid and accumulation of vitelliform material at day 216. c Fundus autofluorescence highlighting the serous detachments and subretinal material at day 216. d Optical coherence tomography (Heidelberg, Germany) reveals presence of subretinal and intraretinal fluid. e-g Subretinal fluid noted at diagnosis remained present at day 117 (e), day 153 (f), and day 216 (g). 